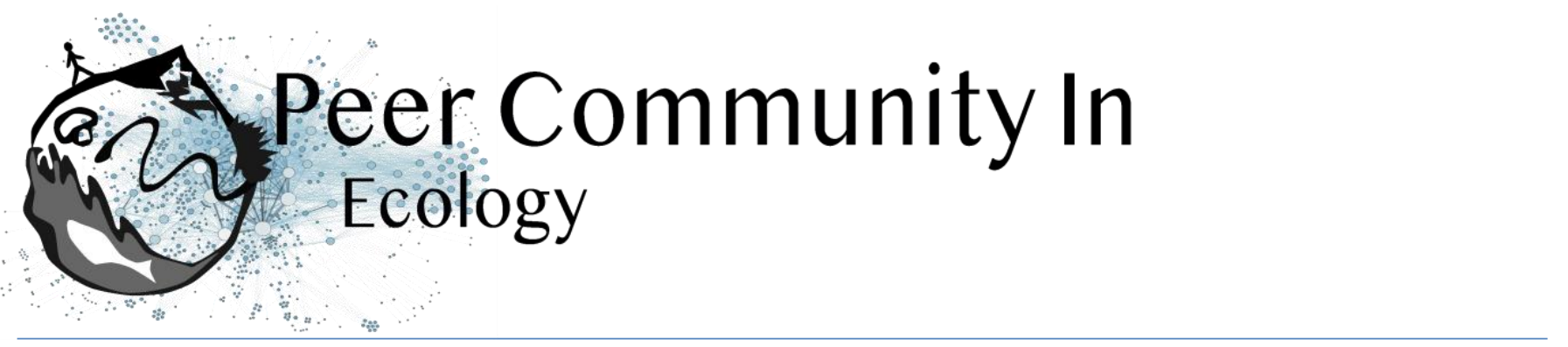

\title{
Does the active vocabulary in Great-tailed Grackles supports their range expansion? New study will find out
}

Jan Oliver Engler based on reviews by Guillermo Fandos and 2 anonymous reviewers

\section{A recommendation of:}

A study on the role of social information sharing leading to range expansion in songbirds with large vocal repertoires: Enhancing our understanding of the Great-Tailed Grackle (Quiscalus mexicanus) alarm call

Samantha Bowser, Maggie MacPherson (2021) OSF, 2UFJ5, version 3, In principle recommendation by $\mathrm{PCI}$ Ecology 10.17605/OSF.IO/2UFJ5

\section{Open Access}

Submitted: 01 December 2020, Recommended: 25 July 2021

\section{Recommendation}

Published: 12 August 2021

Copyright: This work is licensed under the Creative Commons Attribution-NoDerivatives 4.0 International License. To view a copy of this license, visit http://creativecommons.org/licen ses/by-nd/4.0/
Alarm calls are an important acoustic signal that can decide the life or death of an individual. Many birds are able to vary their alarm calls to provide more accurate information on e.g. urgency or even the type of a threatening predator. According to the acoustic adaptation hypothesis, the habitat plays an important role too in how acoustic patterns get transmitted. This is of particular interest for range-expanding species that will face new environmental conditions along the leading edge. One could hypothesize that the alarm call repertoire of a species could increase in newly founded ranges to incorporate new habitats and threats individuals might face. Hence selection for a larger active vocabulary might be beneficial for new colonizers. Using the Great-Tailed Grackle (Quiscalus mexicanus) as a model species, Samantha Bowser from Arizona State University and Maggie MacPherson from Louisiana State University want to find out exactly that.

The Great-Tailed Grackle is an appropriate species given its high vocal diversity. Also, the species consists of different subspecies that show range expansions along the northern range edge yet to a varying degree. Using vocal experiments and field recordings the researchers have a high potential to understand more about the acoustic adaptation hypothesis within a range dynamic process.

Over the course of this assessment, the authors incorporated the comments made by two reviewers into a strong revision of their research plans. With that being said, the few additional comments made by one of the initial reviewers round up the current stage this interesting research project is in. 


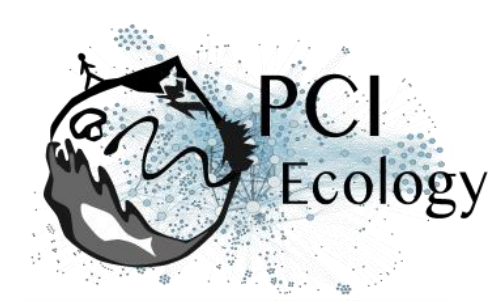

To this end, I can only fully recommend the revised research plan and am much looking forward to the outcomes from the author's experiments, modeling, and field data. With the suggestions being made at such an early stage I firmly believe that the final outcome will be highly interesting not only to an ornithological readership but to every ecologist and biogeographer interested in drivers of range dynamic processes.

\section{References}

Bowser, S., MacPherson, M. (2021). A study on the role of social information sharing leading to range expansion in songbirds with large vocal repertoires: Enhancing our understanding of the Great-Tailed Grackle (Quiscalus mexicanus) alarm call. In principle recommendation by PCI Ecology. https://doi.org/10.17605/OSF.IO/2UFJ5. Version 3

\section{Cite this recommendation as:}

Jan Oliver Engler (2021) Does the active vocabulary in Great-tailed Grackles supports their range expansion? New study will find out. Peer Community in Ecology, 100087. 10.24072/pci.ecology.100087

\section{Reviews}

\section{Reviewed by anonymous reviewer, 2021-06-18 17:45}

I reviewed the first version of this outline. The authors have done an excellent job addressing all the previous reviewer comments, and I think the outline has improved substantially as a result. I really appreciate how the authors rewrite and refine some of the predictions, and the incorporation of much more detail along with all the manuscript (e.g. background point selection, or the subsection "training")

\section{Minor comments}

I think that incorporating the phylogenetic with the PPCA and PGLS will help to disentangle if the differences in vocalisations are more or less than expected the phylogeny. Good job here.

For the ensemble distribution models, what algorithms do you plan to use? Do you consider applying a single model approach if one of the models outstand the others?

I agree that considering the group size could tackle the problem. But do you think that vocal call variability and responsiveness could be density-dependent? In that sense, a value of bird density would be a variable to include in the analysis.

Maybe it is interesting to analyse if the non-vocal displays and vocal displays are negatively correlated. Subspecies might have differences in how they respond, showing how species can develop differing adaptations for biotic interactions (Jedlikowski et al. 2021).

As an alternative or to complement the AUC values to assess the accuracy of species distribution models, you can use Kappa and the True Skill Statistic (TSS).

\section{Revision round \#1}




\section{(4.7. $\mathrm{PCl}$. Ecogy}

\section{Author's Reply}

Download author's reply (PDF file)Download tracked changes file Thank you for your patience! :)

\section{Decision round \#1}

Dear authors,

This is my first contribution handled through $\mathrm{PCl}$, please apology the long handling time while figuring out how the system and the ideas behind PCI fully flesh out.

At first I was quite surprised to see that this is not a preprint per se but rather an outline of what eventually will become a nice study. I got thrilled on it as I really like the idea to embark an external board of reviewer that accompany a project already at the early stages. I think this is tremendously helpful, especially if one is trying to investigate new questions or using unfamiliar tools. It means more work for us though as I am now expecting an iterative process where the same team of reviewer will revisit your work to see how you have been doing. So it's far from a simple review what I was initially expecting but is rather a journey we take together - which I actually like the more I think about it.

With all that being said, you will find enclosed two reviews of two highly trusted peers of mine and I think they provide really good feedback what will help your envisioned research. Even though there might be some "make it or break it" issues I think they could be of help to navigate around them at that early stage of your work. E.g. considering some simulation work to frame null models against which your findings could be compared (like in anything related to phylogenetic relationships/distances).

I hope you will be able to answer the excellent points provided by the reviewers.

All the best

Jan

\section{Preprint DOI: https://doi.org/10.17605/OSF.IO/2UFJ5}

\section{Reviewed by Guillermo Fandos, 2021-03-01 12:59}

Bird calls may provide crucial information on the effects of geographic isolation between subspecies or populations and may reflect local adaptation to diverging ecological conditions. Studies of variation in learned vocalisations are a promising complementary tool to traditional ones for identifying potential dispersal barriers. To this end, the authors provide a welldesigned and hypotheses-driven approach that could yield exciting insights into the mechanisms that govern range expansion in Q. mexicanus subspecies. I find it very interesting. Nevertheless, I have some recommendations and concerns about the methods and statistical analysis to fully use the exciting outcomes.

First, in oscine Passerines, learning influence vocal development. Thus, vocal variation may be assigned to cultural differences that occur among different populations. The wide range of vocalisation in Q. mexicanus is likely the evolutionary result of the intense courtship and sexual selection behaviours. However, alarm calls development could be driven by different selective pressures that determinate a homogenisation between subspecies. For instance, the similarity 
in alarm call structure among species and subspecies might facilitate predator detection. I find that the manuscript will benefit from further explaining the implications of analysing alarm calls and not other social calls that could drive a better picture of how social communication drives differences in the range expansion.

Second, although authors already state that disturbed habitats could influence the range expansion in some of their hypotheses, it would be necessary to provide further background and discussion about the role of urbanisation (as specific disturbed habitat) in these range expansion differences heterogenous intraspecific social communication. Some studies point out the importance of urbanisation in explaining the northward expansion differences between subspecies (Christensen, 2000; Wehtje, 2003). In that sense, urbanisation can influence range expansion, and at the same time, drive differences in vocal communication (high degree of behavioural flexibility) since they are found at much higher densities in urban areas than anywhere else, less predation and higher availability of food resources (Christensen, 2000). I suggest to the authors to include urbanisation variables to control differences in the alarm calls and in the ensemble distribution modelling approach and discuss further the urbanisation role in their hypothesis.

Third, I find a lack of detail in the ensemble distribution modelling approach. Probably, authors will need another step and metrics for niche comparison between subspecies (e.g. niche overlap; Broennimann et al. 2012). In that sense, how they are planning to include the background information in the modelling approach, what algorithms they are planning to use? For instance, a specific background for each subspecies (this could result in an overfitting model for each subspecies). Besides, niche models and differences between subspecies niches could be influenced by different sampling effort, and observation bias between subspecies ranges that could contain eBird data. Western states are better sampled and contain more complete lists than Central USA. Following similar reasoning, differences between generalist and specialist could be drawn by limitations and sampling biases (different realised niche between subspecies but the same fundamental niche).

Finally, I found a lack of explanation of the integration between the alarm call experiment and niche modelling approach. How will be tested if alarm call heterogeneity is correlated with niche differences or heterogeneous landscape? Maybe further integration with other tools (e.g. genetic).

Specific comments

While I am no expert about call analysis, authors should include information about the microphone distance to the target individual since it could influence some of the experiment's metrics.

- Could the close geographical distance of the proposed research areas from Q. m. nelson and Q. m. monsoni influence the playback experiment?

- What the authors mean with "near wetlands" in the statement of the Alternative $1 \mathrm{~b}$ hypothesis?. Do you plan to draw a buffer around the wetlands? In that sense, it would be necessary to include some dispersal information.

\section{References}


- Broennimann, O., Fitzpatrick, M. C., Pearman, P. B., Petitpierre, B., Pellissier, L., Yoccoz, N. G., ... \& Guisan, A. (2012). Measuring ecological niche overlap from occurrence and spatial environmental data. Global ecology and biogeography, 21(4), 481-497.

- Christensen, A. F. (2000). The fifteenth- and twentieth-century colonization of the basin of Mex-ico by the great-tailed grackle (Quiscalus mexicanus). Global Ecology and Biogeography, 9(5), 415-420.

- Wehtje, W. (2003) The range expansion of the great-tailed grackle (Quiscalus mexicanus Gmelin) in North America since 1880. Journal of Biogeography, 30(10), 1593-1607.

\section{Reviewed by anonymous reviewer, 2021-02-05 11:27}

\section{Dear authors,}

You deal with an interesting study topic. Range extensions certainly deserve more attention and I like your very integrative approach. Your study concept seems to be thoughtful and well organized. I mainly have some smaller concerns but I assume that you uploaded your concept to PCI Ecology in order to look for an "advocatus diaboli". Hence, I also want to stress some larger concerns dealing with study design in general. From my point of view this is not only a very interesting experimental study dealing with plenty of possible predictors and pretty rigorous hypothesis testing but also a comparative study. 1) Comparative studies deal conceptually with coalescence and are typically not feasible with such a small number of species/taxa. Differences among the three subspecies could be based on their common ancestry, i.e. two taxa are almost certainly more closely related to each other than to the third group. Any differences among them could be "simply" related to their past history. Typical comparative studies deal with rather taxon-rich phylogenies in order to figure out evolutionary driving forces. It seems hard to study this in a three-taxa system. I am fully aware that literature is full of comparisons among few (perhaps even between two) species (or subspecies or populations) and that far-ranging conclusions are inferred from this kind of studies but actually, great caution is warranted. I am not sure that you will be able to fully reject any null hypotheses if one keeps this in mind. This also relates to my next concern.

2) You look for differences among three groups but these groups are spatially clustered - the three taxa occur (necessarily for subspecies) in three different geographical areas. Although you check for biological meaningful variables associated with different areas (habitat), this does not exclude any other random factor associated with a west-east gradient (e.g. Iongitude per se or even any other absurd factor could not be excluded due to inherent problems of study design). This argument is similar to the first concern. I do not know if you follow my argumentation. The problem is that there is no true solution for these issues (theoretically, you could check for relationships among subspecies) but you should at least be aware of the problem. I have some additional minor concerns. It should be more clearly stated if you interpret possible differences in bioacoustics as consequence of different social behavior or as plastic (or evolutionary) consequence of to the environment. As far as I understood, you tackle all these aspects but this needs to be distinguished right at the beginning of the outline. Note, that calls are generally believed to be genetically determined (I do not know if this has been shown in your study species, too?). Note also, that any differences in call parameters can be simply due to chance (e.g. based on a Brownian motion model often assumed in character evolution and actually a kind of null model against one should test evolutionary changes, see main concern 1 above). I am pretty sure that you are aware of this and playback experiments 


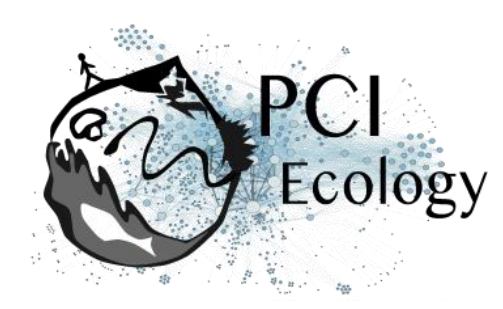

are a great idea to tackle this issue. Also note that there are size differences among subspecies. Song frequency is necessarily heavily influenced by size and differences in pitch can be simply based on size (environmental or social factors might be actually irrelevant in this case).

Details Abstract Q. m. monsoni in italics

4 Hypotheses Alternative 1a Habitat availability is certainly a very crucial factor. Keep in mind that you have to consider the area reachable for the birds in a given time. The delimitation of the background is very important for any spatial modelling and these considerations could be added to your outline.

7 Data collection procedures I highly recommend some training, keeping bird in your view (and record age+sex) + recording of background noise + actually recording and subsequently performing your experiment + covariates like weather etc. is not easy. I am pretty sure you already know that :-) You use many covariates and this is certainly warranted. However, it seems reasonable to limit experiments and recordings to suitable weather conditions (no storm, no rain). For playback experiments always use uncompressed files (e.g. wav) as birds might not react to compressed mp3-files (I am sure you are aware of this, too but I once made this mistake myself when trying to map nightjars).

10 Stopping rule Your power analysis seems good to me but you also mention a few drawbacks. You are dealing with field data that only later might have to be excluded (e.g. because problems with the recording not immediately obvious). So, in practice, always add a few more recordings. Actually, the sample size is very ambitious, anyway! You should expect differences in sexes (e.g. due to size differences) and while you consider sex as a demographic variable you might have to make your analyses for males and females separately. Not that differences among sexes could be specific for each subspecies making comparisons particularly difficult. 12 Measured variables Note that AUC has been heavily criticized in the literature (e.g. Lobo et al. 2008, Global Ecol. Biogeogr.). It might make sense to add an additional value for model evaluation (e.g. Kappa). Variable contribution is a big task in spatial modelling. I am not familiar with the approach by Curry et al. 2018 but most approaches (like Maxent) are not built for this task Which scale do you chose for co-occurrence? Not a very big problem but you should select an appropriate pixel/grid cell size for co-occurrence, e.g. do birds that occur in the same $1 \times 1,5 \times 5$ or $10 \times 10 \mathrm{~km}$ grid actually influence each other (the smaller the better)? 15 Blinding What about the third population? I am a bit nitpicking but do not name the subspecies according to US federal states. The birds are more widely distributed (e.g. in Mexico) and for outsiders this is more difficult to follow than scientific names. 18 Ensemble Distribution Models Did you consider methodological alternatives? As far as I understood Curry et al. 2018 is not a typical ensemble approach as they do not rely on different modelling algorithms like e.g. Biomod. I do not want to criticize Curry et al. 2018 but recommend selecting the approach most suitable for your data and Biomod or Maxent might be alternatives (which you might simply reject for a good reason). Given that modelling is very complex, this aspect of your outline could be a bit more detailed.

I look forward to see your first results being published.

Kind regards 